\title{
Sensitivity of Type I X-Ray Bursts to rp-Process Reaction Rates
}

\author{
A Matthew Amthor ${ }^{* a b c d}$, Daniel Galaviz ${ }^{a b}$, Alexander Heger ${ }^{b d e}$, Alexander \\ Sakharuk $^{a b}$, Hendrik Schatz ${ }^{a b c}$, Karl Smith ${ }^{a b c}$ \\ ${ }^{a}$ National Superconducting Cyclotron Laboratory, Michigan State University \\ 1 Cyclotron; MSU; East Lansing, Michigan 48824-1321; USA \\ ${ }^{b}$ Joint Institute for Nuclear Astrophysics (JINA) \\ ${ }^{c}$ Department of Physics and Astronomy, Michigan State University \\ East Lansing, Michigan 48824-1116; USA \\ ${ }^{d}$ Theoretical Astrophysics Division, Los Alamos National Laboratory \\ MS B227; Los Alamos, New Mexico 87545; USA \\ ${ }^{e}$ Department of Astronomy and Astrophysics, University of California, Santa Cruz \\ Santa Cruz, California 95064; USA
}

First steps have been taken in a more comprehensive study of the dependence of observables in Type I X-ray bursts on uncertain $(\mathrm{p}, \gamma)$ reaction rates along the $r p$-process path. We use the multizone hydrodynamics code KEPLER which implicitly couples a full nuclear reaction network of more than 1000 isotopes, as needed, to follow structure and evolution of the X-ray burst layer and its ashes. This allows us to incorporate the full $r p$-process network, including all relevant nuclear reactions, and individually study changes in the X-ray burst light curves when modifying selected key nuclear reaction rates. In this work we considered all possible proton captures to nuclei with $10<Z<28$ and $N \leq Z$. When varying individual reaction rates within a symmetric full width uncertainty of a factor of $10^{4}$, early results for some rates show changes in the burst light curve as large as 10 percent of peak luminosity. This change is large enough to be detectable by current X-ray burst light curve observations. More precise reaction rates are therefore needed to test current X-ray burst models, particularly of the burst rise, with observational data and to constrain astrophysical parameters.

International Symposium on Nuclear Astrophysics - Nuclei in the Cosmos - IX

25-30 June 2006

CERN, Geneva, Switzerland

\footnotetext{
*Speaker.
} 


\section{Introduction}

Type I X-ray bursts are triggered by a thermonuclear runaway in a thin layer of material accreted from a companion star. They occur for certain accretion rates at which the hydrogen and helium nuclear fuel are largely consumed through the rapid proton capture process ( $r p$-process) and the $\alpha$ p-process, respectively. The rp-process takes place under conditions of extreme temperature and density in regions of proton excess and thus functions as a series of fast proton captures and weak decays involving isotopes on the proton-rich side of stability, including several near the proton drip-line. The process is therefore sensitive to nuclear structure properties far from stability, mostly unknown to nuclear physics.

Multi-zone burst models allow the study of the evolution of the neutron star crust composition over time, from accretion through stable burning, through the burst, and following the burst. This allows consideration of effects which are impossible to study using only single-zone models. Through compositional inertia, the ashes of earlier bursts affect the character of later ones. Also, the reheating of burst ashes by successive bursts will further process the nuclear material. The resulting abundances in deeper layers of the neutron star crust, specifically that of carbon, are relevant to superburst processes $[1,2]$.

This work examines the sensitivity of Type I X-ray bursts to all possible proton captures (those which produce particle stable compound systems) on nuclei with $10<Z<28$ and $N \leq Z$, varying one rate at a time within an assumed uncertainty, with the goal of determining the extent to which our ability to model these systems is limited by our current knowledge of the nuclear physics inputs. Similar studies conducted previously used postprocessing techniques [3] or analytical methods [4] in single-zone models, but because the rp-process and $\alpha$ p-process to be studied are the main energy sources, such a treatment is inadequate here. We used a specially adjusted, single-zone burst model to make a rough search for sensitivity in the burst light curve and nucleosynthesis in the full set of reactions. These results were compared, in selected cases, to the results of more rigorous multizone simulations.

\section{Reaction Rates}

Hundreds of uncertain proton capture rates lie on the $r p$-process path and can individually or collectively influence the energy generation and/or nucleosynthesis that will take place during a burst. Direct measurement of these reaction rates is difficult [5]. Unknown compound reaction rates are frequently estimated using statistical model calculations. These calculations, however, are only valid for capture to a region of sufficiently high level-density in the compound nucleus. At burst temperatures this condition is often not satisfied for proton capture on lighter nuclei, $Z \lesssim 50$, near the proton drip line [6]. Proton capture rates to compound systems with low level density near and above the proton threshold will be dominated, at burst temperatures, by a few resonant capture contributions. Therefore, the properties of these lowest energy proton-unbound levels critically determine the capture rates.

In the absence of experimental structure information on such isotopes along the rp-process path, shell model calculations are used, sometimes together with information from the mirror nucleus. The commonly assumed $100 \mathrm{keV}$ uncertainty in the excitation energy of theoretically cal- 


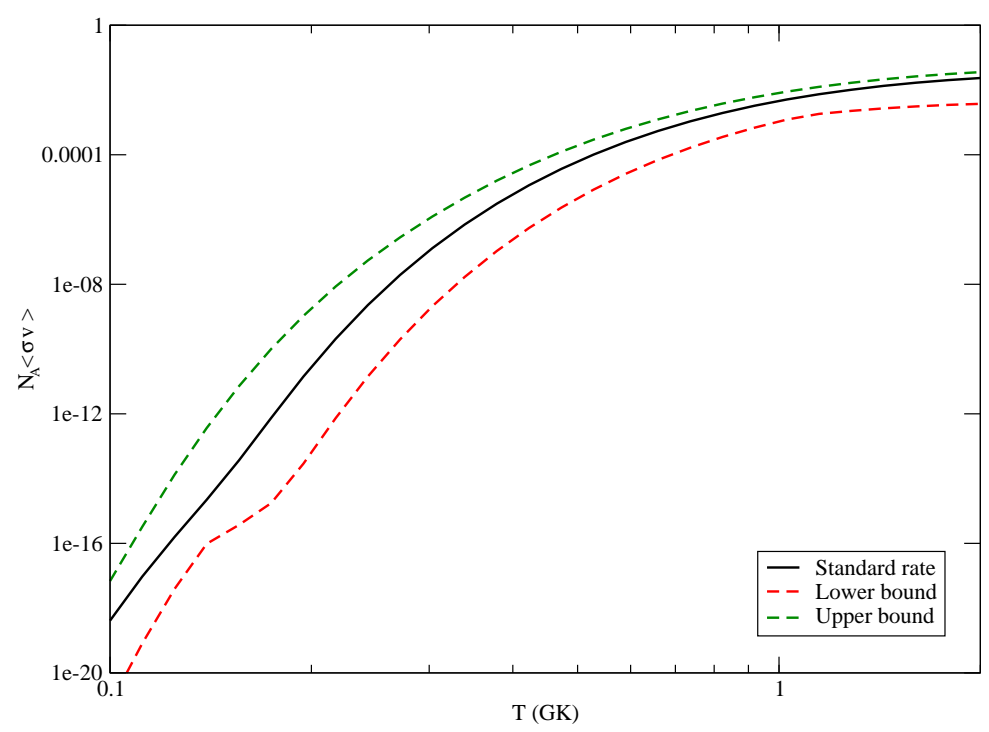

Figure 1: Shown is the calculated uncertainty for the ${ }^{42} \mathrm{Ti}(\mathrm{p}, \gamma)^{43} \mathrm{~V}$ reaction rate, assuming $100 \mathrm{keV}$ uncertainty in resonance energies.

culated levels in these cases can yield orders of magnitude uncertainty in the reaction rates for temperatures relevant in Type I X-ray bursts. For example, Figure 1 shows the rate and uncertainty for the ${ }^{42} \mathrm{Ti}(\mathrm{p}, \gamma)^{43} \mathrm{~V}$ reaction, calculated using structure information from Herndl et al. [7]. These calculated uncertainties have been shown to be reasonable in recent experiments $[8,9]$. In the burst simulations of the present study, we assume that such a situation is typical and adopt a temperature-independent, symmetric uncertainty with a full-width of a factor of $10^{4}$.

\section{Single-zone model}

One-dimensional, multi-zone models are too computationally expensive to consider all possible rate uncertainties; so we begin with single-zone burst simulations. The single-zone model of Schatz et al. [10] was used, taking abundances and thermodynamic conditions from the burst zones in KEPLER [1] as initial conditions. The specific zone and initial conditions used were adjusted in order to best reproduce the lightcurve and final abundances seen in the multi-zone model when using reaction rates unchanged from the current rate library. It is perhaps worth noting that the reaction rate library being used by the single-zone model [11] is not exactly the same as that used by KePLER [1].

The process of running many single-zone simulations with changes to specific proton capture rates was automated, and each burst simulation took approximately $1.5 \mathrm{cpu}$ minutes. Very few of the initially considered rate variations caused a significant change in the burst lightcurve, but the changes that were observed were not subtle. The changes seen in the burst ashes were generally less pronounced with a more continuous distribution of standard deviations. In general, the lower bound reaction rates showed fewer and less significant changes from the baseline in both the lightcurves and the burst ashes than did the upper bound rates. There is little initially apparent correlation between changes to lightcurves and to the ashes produced. 
In cases where significant changes were observed, it was then more carefully considered to what extent the assumed uncertainty was justified for the specific reaction. One of the most dramatic changes was seen when increasing the ${ }^{26} \mathrm{P}(\mathrm{p}, \gamma){ }^{27} \mathrm{~S}$ rate. But since ${ }^{27} \mathrm{~S}$ has a proton separation energy, estimated by systematics, of $800 \mathrm{keV}$ and the lowest excited state in the mirror nucleus is at $1720 \mathrm{keV}$, it is unlikely that the capture on ${ }^{26} \mathrm{P}$ is dominated by uncertain resonant contributions. This rate may still be a controlling factor, but should be varied over a smaller uncertainty. Shell model calculations of the compound system could give us a clearer picture. The rates for

${ }^{46} \mathrm{Cr}(\mathrm{p}, \gamma){ }^{47} \mathrm{Mn}$ and ${ }^{49} \mathrm{Fe}(\mathrm{p}, \gamma){ }^{50} \mathrm{Co}$ on the other hand, are likely dominated by resonant contributions from capture to poorly known levels in the compound nucleus, based on the structure of their respective mirror nuclei. When increased, these rates both produce brighter bursts with shorter risetimes in the single-zone model.

In addition to individual rate variations considered here, higher-order effects should also be considered, as the sensitivity of the process to one rate may be strongly affected by the value of another uncertain rate or set of rates. Naturally, this leads to a much larger sample space which grows both exponentially with order and geometrically with the number of reactions considered. Monte Carlo sampling methods, such as those used for novae in [3], could provide some valuable first insight into the broader set of these high-order dependencies.

\section{KEPLER calculations}

The model used was that with solar metallicity and an accretion rate $\dot{M}=1.75 \times 10^{-9} M_{\odot} y r^{-1}$, model $Z M$ as discussed in [1]. The first burst results from accretion to a bare iron surface and is therefore uncharacteristic of usually observed bursting behavior. We look instead at the third burst, which, for standard reaction rates, shows properties very similar to those of the steady state behavior reached after a longer chain of bursts. Of the several rates to which the single-zone model showed sensitivity, and for which the assumed uncertainty was well justified, those considered so far in the multi-zone model include the upper limit rates for ${ }^{42} \mathrm{Ti}(\mathrm{p}, \gamma){ }^{43} \mathrm{~V},{ }^{46} \mathrm{Cr}(\mathrm{p}, \gamma){ }^{47} \mathrm{Mn}$, and ${ }^{49} \mathrm{Fe}(\mathrm{p}, \gamma){ }^{50} \mathrm{Co}$. The lower limit rate for ${ }^{46} \mathrm{Cr}(\mathrm{p}, \gamma){ }^{47} \mathrm{Mn}$ was also considered.

In the case of an increased ${ }^{46} \mathrm{Cr}(\mathrm{p}, \gamma)$ rate, we observe a similar large effect on the third burst in the multi-zone model to that seen in the single-zone model (see Figure 2). For this particular reaction the single-zone model appears well calibrated. This supports the conclusion that burst lightcurves are strongly dependent on this individual reaction rate. In the case of ${ }^{42} \mathrm{Ti}(\mathrm{p}, \gamma)$, there are still slight differences in the decay of the lightcurve, but in the multi-zone model the increased rate results in faster cooling, the opposite of the effect seen in the single-zone model. Also, the multi-zone simulations show faster rise and higher peak luminosity for this upper-limit rate in the second and third burst, which were not seen in the single-zone model. In the case of ${ }^{49} \mathrm{Fe}(\mathrm{p}, \gamma)$, the multi-zone model shows only a very slight increase in peak luminosity of the third burst, while the single-zone model showed an increase of over five percent in peak luminosity and a risetime $\approx 500$ ms faster.

Clearly, a one zone model cannot be expected to correlate exactly with the results of a multizone simulation, especially during risetime, when convection plays an important role. Nevertheless, this strategy can serve as an initial guide to help narrow the list of potentially important reaction rates. It should be noted that, although the third burst seems fairly well converged, the studies of 


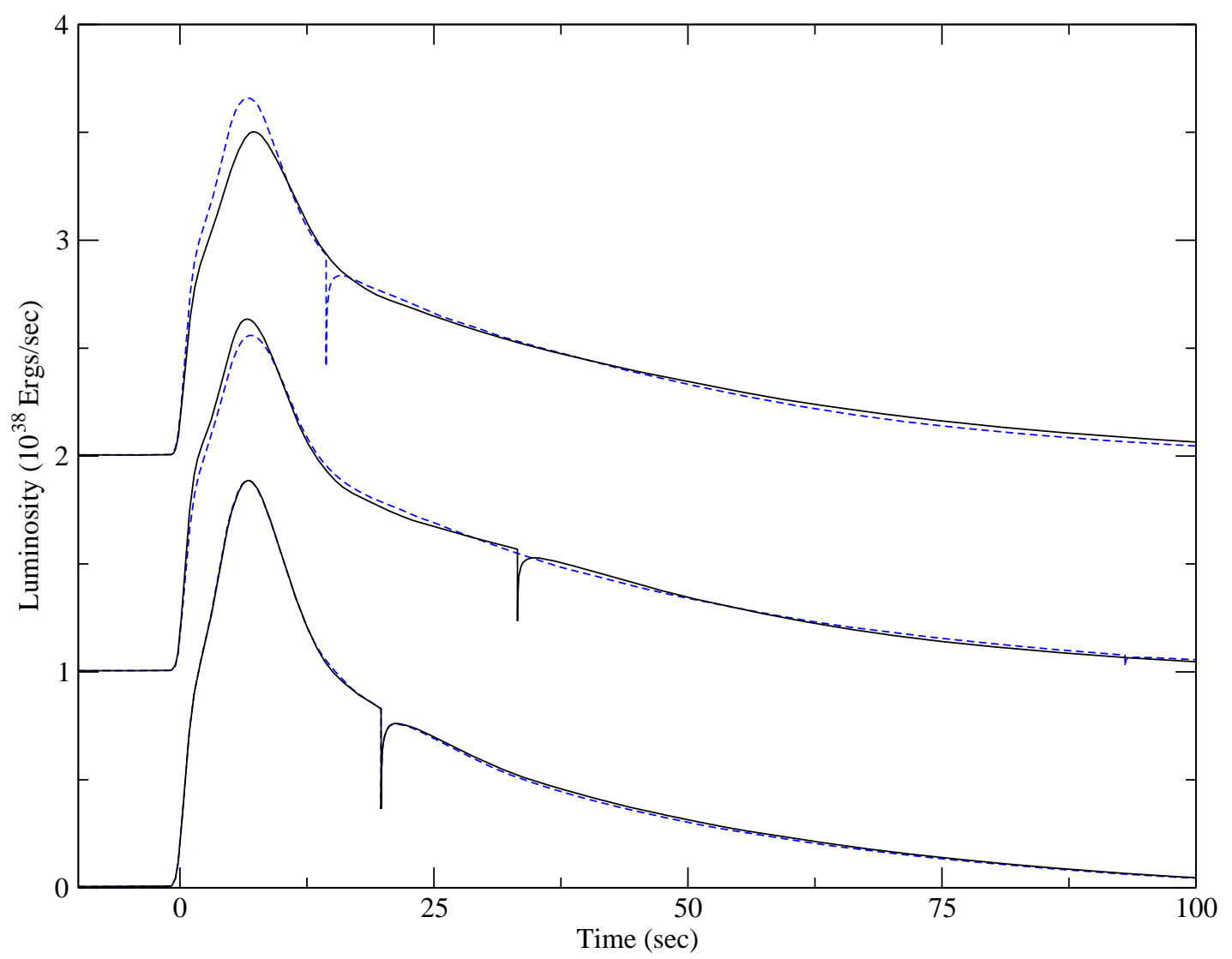

Figure 2: Bursts one, two and three for the solar metallicity, high accretion rate model (model ZM in [1]) in the KEPLER code are shown, with later bursts shown successively higher on the graph. Standard reaction rates yield the solid curves and an increased ${ }^{46} \mathrm{Cr}(\mathrm{p}, \gamma)$ rate yields the dashed curves. In the third burst the increased rate results in an increase of over 10\% in peak burst luminosity. The small dips are the result of a numerical artifact in the code.

all rate sensitivities should be continued until a steady state behavior is reached, and ideally the exact same rate library should be used for both models to eliminate this as a possible source for discrepancies.

\section{Conclusions}

Based on the few reactions studied so far it seems that the one-zone approximation can help to identify critical rates in some cases. While there can also be large differences in the sensitivities to rates between the two models, the one-zone approximation can be used as an initial guide to narrow down the list of potentially important rates to be studied with the multi-zone model. This approach does not necessarily identify all rate sensitivities, but within the computing time limits it provides an effective way to determine some of them. This observation further demonstrates the importance of considering effects which exist only in higher dimensions and will hopefully encourage work on codes that can consider Type I bursts in greater than one dimension. In the future, the one-zone model analysis could be extended to the variation of multiple rates or to a full Monte Carlo analysis. 
There is at least one case found in this preliminary analysis with good evidence of a strong dependence of the burst lightcurve on a single reaction rate. The other uncertain reaction rates considered in the multi-zone code to date appear not to be such critical inputs to the process for the specific accretion model considered.

\section{Acknowledgments}

This work was carried out under the auspices of the National Nuclear Security Administration of the U.S. Department of Energy at Los Alamos National Laboratory under Contract No. DEAC52-06NA25396, and was also supported by the U.S. National Science Foundation Grants No. PHY-01-10253 (NSCL) and No. PHY-02-016783 (JINA).

\section{References}

[1] S. E. Woosley et al., Models for Type I X-Ray Bursts with Improved Nuclear Physics, Astrophysical Journal Supplement Series 151 (2004) 75.

[2] R. E. Taam, X-Ray Bursts from Thermonuclear Runaways on Accreting Neutron Stars, The Astrophysical Journal 241 (1980) 358.

[3] W. R. Hix, M. S. Smith, S. Starrfi eld, A. Mezzacappa and D. L. Smith, Impact of nuclear reaction rate uncertainties on Nova models, Nuclear Physics A 718, 620 (2003)

[4] R. G. Izzard, M. Lugaro, C. Iliadis, A. Karakas, Reaction Rate Uncertainties: NeNa and MgAl in AGB Stars, these Proceedings

[5] S. Bishop et al., ${ }^{21} \mathrm{Na}(p, \gamma)^{22} \mathrm{Mg}$ Reaction and Oxygen-Neon Novae, Physical Review Letters 90 , $162501(2003)$

[6] T. Rauscher, F.-K. Thielemann and K. L. Kratz, Nuclear level density and the determination of thermonuclear rates for astrophysics, Physical Review C 56, 1613 (1997)

[7] H. Herndl, J. Görres, M. Wiescher, B. A. Brown, and L. Van Wormer, Proton capture reaction rates in the rp process, Physical Review C 52, 1078-1094 (1995)

[8] R. R. C. Clement et al., New Approach for Measuring Properties of rp-Process Nuclei, Physical Review Letters 92 (2004) 172502.

[9] D. Galaviz et al., High precision measurements along the rp-process path, these Proceedings

[10] H. Schatz et al., End Point of the rp Process on Accreting Neutron Stars, Physical Review Letters 86 (2001) 3471.

[11] A. Sakharuk et al., An Updated Library of Reaction Rates for the Astrophysical rp-Process, $12^{\text {th }}$ International Symposium on Capture Gamma-Ray Spectroscopy and Related Topics, Notre Dame, Indiana, September 2005. AIP Conference Proceedings, Vol. 819. Edited by Andreas Woehr and Ani Aprahamian. Melville, NY: American Institute of Physics, 2006., p.118-122 